\title{
Cibercultura, internet y salud móvil
}

\author{
Cyberculture, internet and health mobile
}

Rodolfo Rodríguez-Gómez¹ orcid.org/0000-0001-5873-4312

1 Freelance como Asesor metodológico de investigación. Bogotá, Colombia

\section{Resumen}

Introducción: A la luz de las nuevas tecnologías, el área de la salud se enfrenta a nuevos escenarios que están transformando el mundo, uno de estos es el de la salud móvil que ha pasado de ser una utopía a una realidad. Para lograr conocer los ámbitos de la salud electrónica y la salud móvil, es imprescindible comprender el trasfondo en el que estos avances se desarrollan. Dicho contexto se enmarca en los terrenos de Internet, la cibercultura y la inteligencia colectiva, los cuales se construyen sobre las bases de la complejidad de la acción y la interacción humana. Objetivo: Analizar la trascendencia de conceptos clave para entender el trasfondo de la salud móvil. Materiales y métodos: Revisión documental de textos físicos y bases de datos electrónicas. Resultados: Se estructuró la información en tres categorías: cultura, ciberespacio e inteligencia colectiva; acción, interacción e Internet, y por último; salud móvil y Apps. Conclusiones: Para la sociedad actual de la información y el conocimiento, áreas de vanguardia como Internet y los dispositivos móviles son más que una tendencia tecnológica; se han convertido en ejes de innovación y construcción de futuro. La tecnología ofrece posibilidades fascinantes, sin embargo, es importante encontrar un sentido más profundo en desarrollos científicos que pueden cambiar el mundo como la salud móvil.

Palabras clave: Internet; tecnología; comunidad; salud; tecnología de la información. (Fuente: DeCS, Bireme).

\begin{abstract}
Introduction: In light of new technologies, the health area faces new scenarios that are transforming the world. One of these is the mobile health that has gone from being a utopia to a reality. To get to know the areas of electronic health and mobile health, it is essential to understand the background in which these advances are developed. This context is framed in the Internet, cyberculture and collective intelligence which are built on the basis of the complexity of action and human interaction. Objective: To analyze the transcendence of key concepts to understand the underlying of mobile health. Materials and methods: A documentary review of physical texts and electronic databases was made. Results: The information was structured in three categories: the first one was culture, cyberspace and collective intelligence; the second one was action, interaction and the Internet, and the last one was mobile health and apps. Conclusions: For the current society of information and knowledge, cutting-edge areas such as the Internet and mobile devices are more than a technological trend. They have become axes of innovation and construction of the future. Technology offers fascinating possibilities; however, it is important to find a deeper sense of scientific developments that can change the world as mobile health.
\end{abstract}

Keywords: internet; technology; society; health; information technology. (Source: DeCS, Bireme).

\footnotetext{
*Autor de correspondencia

Rodolfo Rodríguez Gómez

e-mail: epidokto17@gmail.com
} 


\section{Introducción}

En la actualidad el mundo gira en torno a la cultura digital; de hecho, los procesos de comunicación en la sociedad se influencian cada vez más de las Tecnologías de la Información y la Comunicación(1). El área de la salud, que hasta hace pocos años parecía mantener cierta distancia con el ambiente digital, se conecta irremediablemente cada vez más con las lógicas que impregnan esta nueva cultura. Por supuesto, estos procesos no han sucedido de la noche a la mañana y un largo camino se ha recorrido desde la Segunda Guerra Mundial cuando fue diseñada Colossus, la primera computadora programable electrónica en cuyos estudios fue determinante el matemático británico Alan Turing(2). El principio de computabilidad basado en algoritmos fue refinado mediante la introducción de lo que se llamó la máquina de Turing, la cual está en íntima relación con los conceptos de complejidad(3). De esta manera, las acciones de Turing y otros investigadores gestaron eventos que cambiarían el mundo de la computación $\mathrm{y}$, gracias a ello, hoy se los considera como precursores de la informática moderna, la misma que junto con los avances de las Tecnologías de la Información y la Comunicación modifican la sociedad e impactan de manera sustancial en el área de la salud(4). Este artículo, pretende hacer visible la trascendencia de conceptos clave para entender el trasfondo de la salud móvil e incentivar en su reflexión, ya que más allá del uso de la tecnología de manera instrumental, se trata de alcanzar el aspecto esencial dentro de la sociedad, que es comunicar.

\section{Materiales y métodos}

El presente artículo se basó en una amplia búsqueda de documentos relacionados, en especial, en bases de datos electrónicas donde se utilizaron tanto palabras clave como términos libres. Las principales bases de batos consultadas fueron: PubMed, Google Scholar y Google Books. También se consultaron sitios web corporativos e institucionales y blogs especializados de actualización reciente así como textos físicos. Aunque no se aplicó restricción idiomática, la búsqueda se concentró en documentos en inglés y español. Los principales términos de búsqueda en español fueron: cibercultura, ciberespacio, internet, cultura, comunicación, innovación, memética, aplicaciones, salud móvil, inteligencia colectiva, acción, interacción, tecnología, web, entre otros. En inglés fueron: Technology, information, internet, web, society, memetic, mHealth, app, mobile devices, healthcare. A pesar de no establecer límites en cuanto a las fechas, dadas las características del tema, se prestó especial atención en documentos de reciente publicación. Las búsquedas se realizaron entre abril de 2015 y septiembre de 2016 recuperando cerca de 90 documentos considerados potenciales de los cuales 46 se incluyeron como referencias del artículo. La información recopilada fue leída en su totalidad y sus contenidos analizados a fin de extraer conceptos y categorías lo cual permitió estructurar el artículo y, al mismo tiempo, incorporar elementos de la discusión en todo el documento.

\section{Resultados}

Los resultados se presentan en tres acápites principales: el primero explora los diferentes elementos relacionados con la cibercultura, el ciberespacio y la inteligencia colectiva. El segundo se enfoca en la trascendencia de la acción y la interacción en Internet y el tercero, puntualiza lo concerniente a la salud móvil y las Apps.

\section{Cibercultura, ciberespacio e inteligencia colectiva}

Desde los albores de la humanidad las poblaciones han desarrollado diferentes culturas; con el surgimiento de estas, los seres humanos han logrado enfrentar situaciones adversas y satisfacer diversas necesidades(5). Tanto la naturaleza como la cultura humana son información, pero se diferencian en la forma como se transmiten, ya que mientras la información natural se transmite por vía genética, lo cultural se transmite por aprendizaje social y se codifica en el cerebro(5). Por tanto, la noción de naturaleza está relacionada con la esencia, y es la naturaleza humana precisamente, lo que tienen en común los individuos Homo sapiens(6). Pero en medio del nuevo paradigma 
tecnológico, aquella mezcla de naturaleza humana y cultura descubre hoy en día un mundo diferente, el denominado mundo virtual. De esta manera, inmerso en la era digital, el ser humano explora hoy un novedoso campo social donde su naturaleza evoluciona y se adapta a una nueva cultura.

Desde la década de los noventa, tras el arribo de Internet, el escritor y filósofo Pierre Lévy difundió el término cibercultura. Lo planteó como una nueva era de la comunicación donde se acuña un lenguaje dinámico y más universal que el mismo alfabeto, el lenguaje digital(7). Lo interesante es que todo esto gravita en el ciberespacio, que es entendido como un nuevo medio de comunicación que emerge de la interconexión mundial de los ordenadores que hace referencia a un universo de información y, al mismo tiempo, a los seres humanos que navegan por él y lo alimentan(7). La cibercultura, entonces, se desarrolla de manera dinámica y constituye un sistema autoorganizado donde las prácticas culturales se producen de manera permanente(8). En este contexto, es relevante resaltar que más allá de los cambios tecnológicos a los que se enfrenta la sociedad, de Internet emerge una nueva dimensión de la cultura, propia de esta tecnología; producto de una nueva inteligencia social.

La idea de una inteligencia colectiva no es nueva. En el siglo XX, Durkheim señalaba que la misma sociedad constituye una inteligencia que trasciende al individuo( ${ }^{(9)}$. Eran los primeros visos de un concepto que habita la web, ya que los usuarios generan contenido y aprenden unos de otros a mayor velocidad que antes. En 1983, en el libro titulado The Global Brain, el matemático Peter Russell reconocía el potencial de Internet para conectar las mentes en una red global donde la información podía ser compartida con la eficiencia del cerebro humano(10). Hoy es evidente que Internet reinventa el vínculo social en torno al aprendizaje recíproco y por tanto, aquella inteligencia colectiva es ubicua y se coordina en tiempo real(11). Pero Internet es mucho más que ordenadores conectados entre sí, ya que también funciona como una memoria transitiva que se recuerda por las personas(12). Algo similar a la llamada "interdependencia cognitiva en relaciones cercanas"(13), donde en largas relaciones de pareja, cada individuo confía en el otro para que funcione como su banco de recuerdos. Internet entonces, no es un fenómeno banal y no solo consigue cambiar la naturaleza, sino también la velocidad de las relaciones humanas(14).

De la cibercultura emerge un componente de memética que se consolida como gran protagonista en la web. Así como la información genética es imprescindible en la naturaleza humana, también lo es la información cultural que se transmite a través de lo que se denomina meme, es decir, una unidad de información cultural(15). Autores como Knobel y Lankshear, postulan que aquellos memes en la red-imemesse deben considerar como una nueva forma de alfabetización(16). Para otros, como Jean Burguess del Centre for Excellence for Creative Industries and Innovation, los imemes y videos se entienden como la dispersión de ideas replicables expresadas en prácticas(17). Es así, como esas prácticas han hecho extender el uso de Internet y en la actualidad, más de 2.000 millones de personas están conectadas a él; en Colombia los usuarios de dicha tecnología corresponden al $51,7 \%$ de la población(18). Diversas ramas del área de la salud tienen una gran tarea en conocer y entender la cibercultura para lograr aplicarla y es en este terreno donde la salud debe participar al seguir lo que sugieren autores como David Hull quien propone que se debería dejar de hablar de memética y hacer más memética(19).

\section{Acción, interacción e Internet}

Hoy en día gran parte de la acción humana está ligada a las prácticas significantes en Internet y con ello, el mundo es testigo de un abismal cambio cultural y social promovido por las nuevas tecnologías(20). En el ciberespacio, la acción humana al igual que los memes, es replicadora. De allí que la acción en Internet pueda traducirse en viralidad como lo demostró Hotmail años atrás al etiquetar cada correo electrónico con la promoción de un nuevo servicio. Al final, la tasa de crecimiento de Hotmail se convirtió en algo epidémico al crear 
12 millones de nuevos usuarios en sólo 18 meses, lo cual demostró que la información puede ser infecciosa y extenderse de usuario a usuario(21). En otras palabras, muchas acciones en la web involucran la duplicación de un mensaje y esto constituye un verdadero acto de comunicación. Pero es de esto, precisamente, de lo que tanto adolece el área de la salud, en especial la salud pública, ya que es poco lo que se interactúa y por tanto, poco lo que se comunica.

Para el mundo de la web es vital la acción humana la cual se define como todo comportamiento o conducta deliberada(22). Para Marx Weber, una acción era toda conducta en la que el individuo que la produce la establece con un sentido personal es decir, la acción social está referida a la conducta de otros(23). Este fenómeno se extrapola a la web donde lo que se persigue es la interacción. De hecho, aquella acción recíproca que resulta de la acción social privilegia la influencia que ejercen unos sobre otros en una situación de comunicación(24). La interacción es la vida misma de la web 2.0 y por tanto, cada mensaje funciona como un estímulo sobre el destinatario y da lugar a cierta retroalimentación(24), y a que las ideas se autoorganicen de forma espontánea en ausencia de instituciones formales(25). En la web, muchas tendencias emergen de interacciones donde la gente no es consciente que está coordinada, pero funciona para el caso de redes mezcladas de manera homogénea(25). Esto evoca lo que se denomina la 'alquimia de las multitudes'(26) donde lo que modifica Internet es lo que se hace con ella, ya que son los mismos usuarios los que se implican y participan.

La noción de interacción es primordial al pensar en salud electrónica y salud móvil. En Internet, la gente consulta información sobre salud, busca profesionales, visita páginas web institucionales, crea blogs para compartir experiencias en salud, además de que consume y comparte fotos y videos, y se sensibiliza ante la información publicada por otros. Es allí donde el área de la salud, en general, tiene un gran potencial para interactuar, ya que si la gente está conectada, su salud también lo está(27). La clave, entonces, es la interacción porque es ella la que hace evolucionar la red, que para el caso de la web 2.0, se desarrolla en un entorno digital(28). Gracias a este concepto, la popularidad de un contenido web se suele comparar con la extensión de una epidemia donde una red con gran cantidad de conexiones incrementa la exposición(29). De hecho, muchos problemas se pueden modelar como se extienden las epidemias en una red y algunos ejemplos son: la extensión de enfermedades entre la población, la circulación de un chisme en una red social y la diseminación de virus por correo electrónico.

Desde hace algunos años, Internet entró en una nueva dimensión denominada Internet de las cosas. Esta vanguardia digital, plantea que las cosas, es decir, los objetos cotidianos, sean conscientes de su entorno y suban información a la web sin ayuda humana. Es tal el potencial de esta evolución digital que se considera como la Tercera Revolución Industrial(30). Su historia se remonta a 1999 cuando el británico Kevin Ashton propuso el concepto y desde entonces, se plantea que Internet de las cosas tiene el potencial para cambiar el mundo tal y como lo hizo la revolución digital hace unas décadas(31). De hecho, para 2014 se consideraba que cerca de 3.9 millones de cosas estarían conectadas a Internet, pero para 2020, se espera que esa cifra aumente a 25 millones(32). Ante este nuevo panorama digital que representa Internet de las cosas, el área de la salud vivirá una verdadera revolución. Esta dimensión digital abre nuevos horizontes tanto para la atención clínica individual como para otras áreas como la prevención, la promoción de la salud y en general, la epidemiología y la salud pública a través de tecnologías como la salud móvil(33).

\section{Salud móvil y Apps}

La salud móvil, también conocida como mHealth, se define como la práctica de la medicina y la salud pública soportada por dispositivos móviles como teléfonos, dispositivos de monitorización de pacientes, asistentes personales digitales y otros dispositivos inalámbricos(34). Involucra el uso de diversas funciones de telefonía móvil como mensajes de voz y texto, así como otras más complejas como el servicio general de paquetes de radio (GPRS), telecomunicaciones de 
tercera y cuarta generación (3G y 4G), sistemas de posicionamiento global (GPS) y tecnología Bluetooth(35). Actualmente, la salud móvil representa una verdadera oportunidad para ayudar a combatir diversos problemas de salud y las enfermedades(36). En países de bajos y medianos ingresos, los gobiernos han mostrado gran interés en la salud móvil como estrategia complementaria para fortalecer los sistemas de salud y lograr los Objetivos de Desarrollo del Milenio (ODM)(37). Además, la salud móvil involucra una nueva dinámica en áreas como la experiencia del usuario, la relación médicopaciente y por supuesto, el almacenamiento y transmisión de datos lo que permite su uso en tiempo real que constituye, una poderosa herramienta para la epidemiología y la salud pública.

Un aspecto a destacar con la salud móvil es la tendencia a democratizar la salud. Este elemento se hace visible, dado que el uso de dispositivos móviles no discrimina si se trata de un paciente, un profesional sanitario o una institución. De la mano de este concepto está el empoderamiento del paciente, el cual aplica para muchos campos clínicos como la atención a personas de la tercera edad, el cuidado de mujeres embarazadas y los recordatorios para tomar la medicación prescrita, que puede redundar en mejores resultados en salud y mayor eficiencia del sistema de salud(38). De esta manera, empoderar a los pacientes en el autocuidado a través de herramientas digitales representaría una verdadera revolución. A través de diferentes aplicaciones, los pacientes según su enfermedad o condición, cuidan y monitorizan su salud. Por otro lado, se incluye a los cuidadores, familia o grupo de apoyo, un ejemplo son aquellas aplicaciones para pacientes con Alzheimer o demencia que, en caso de que el paciente se aleje de casa, el sistema de GPS dispara una señal que alerta a la familia o el cuidador con lo cual la persona puede ser encontrada y retornada a su entorno de atención( ${ }^{(39) .}$

\section{Discusión}

Para la salud móvil es imprescindible el tema de aplicaciones para dispositivos móviles, las famosas Apps. Actualmente, existen más de 165.000 a nivel mundial(40) y la mayoría están dirigidas al público general en temas como bienestar y ejercicio, mientras que el resto, a profesionales sanitarios y pacientes. Según el Informe de las 50 mejores Apps de salud en español, casi el $50 \%$ de estas son de carácter informativo y para monitorizar parámetros físicos(41), lo que indica ciertas tendencias, nichos de mercado y potenciales áreas de investigación. Según la Food and Drug Administration (FDA), para 2018 el 50\% de los usuarios de teléfonos inteligentes y tabletas habrá descargado una aplicación de salud para dichos dispositivos(42). No obstante, un aspecto de incalculable valor con el uso de Apps en salud es que logran acercar la salud a la gente. Un ejemplo es la Calculadora de Riesgo Cardiovascular que la OPS lanzó en 2014, con motivo del Día Mundial del Corazón, es de carácter gratuito y permite que las personas calculen el riesgo cardiovascular y al mismo tiempo, encuentren consejos sobre autocuidado y pautas para modificar dicho riesgo ${ }^{(43)}$.

Es en este campo del desarrollo de Apps para la salud móvil donde grandes compañías tecnológicas destinan hoy muchos recursos. Uno de los mejores ejemplos es la caja de BlackBerry llamada HealthBox que se desarrolló como una plataforma basada en Internet de las cosas. Este es un servicio de salud donde el paciente se puede mantener siempre en contacto con el médico a través de diferentes dispositivos como una pulsera, un marcapasos o un glucómetro, la información se envía a la nube y de allí, al hospital encargado del tratamiento para ser analizada por el médico(44). Apple ha desarrollado HealthKit, una herramienta tecnológica donde las Apps trabajan para el usuario, administran datos, crean tarjetas con información útil en emergencias e incluso pueden compartir datos como la presión sanguínea con el médico tratante. Para médicos e investigadores, Apple también cuenta con plataformas como ResearchKit que permite a los investigadores recopilar datos para los estudios y CareKit es una plataforma donde se pueden crear Apps que ayuden a las personas a mejorar el autocuidado(45). 


\section{Conclusiones}

El mundo en la era digital requiere de una nueva visión para adaptarse a ella. Para los profesionales del área de la salud, el elemento clave es entender que se viven otros tiempos y más importante aún, que se puede ser protagonista. De esta manera, la salud podrá participar en un nuevo contexto dado por la civilización digital. Este es un gran espacio de intercambio cultural donde emergen la interacción y la cooperación y se gestan nuevas formas de encuentro para participar $y$ comunicar(46). Es un nuevo territorio sin fronteras visibles, donde muchos se han adaptado y han sacado años luz de ventaja, pero otros, hasta ahora se percatan que ese mundo existe. El área de la salud debe levantar la mirada para apreciar todo lo que el mundo virtual ofrece, ya que este nuevo desafío no se trata de un nuevo microorganismo, ni de un brote o epidemia, se trata de algo diferente, pero con una complejidad enorme donde la clave es aprender a interactuar con la comunidad a través de nuevos vehículos y herramientas tecnológicas.

El mundo de Internet, la salud móvil y las Apps son parte del engranaje de una nueva ola de innovación en salud. Esta nueva senda tecnológica está encaminada a trascender el uso meramente instrumental de la tecnología. Se trata, de acoplar cada vez más la tecnología a la cotidianidad, a la vida misma, e incluso a la naturaleza humana. Gracias a diferentes avances como Internet, la inteligencia artificial, la biotecnología, Big data y la nanotecnología, la medicina está en la apertura de caminos nunca antes imaginados y en íntimo diálogo con otras áreas del conocimiento. Sin embargo, todos los avances de la medicina, ya sean clínicos o tecnológicos, tienen un único fin que es mejorar la calidad de vida de la población al interactuar con los pacientes o los potenciales pacientes y alcanzar el objetivo primordial de esa interacción con la sociedad que es la comunicación.

\section{Conflicto de intereses}

Ninguno declarado por los autores.

\section{Referencias}

1. Mincultura. Cultura Digital. [Internet] Bogotá, Colombia: Mincultura; 2015 [cited 2016 Junio 15]; Disponible en: http://www.mincultura.gov.co/areas/comunicacion es/cultura-digital/Paginas/default.aspx

2. Copeland J. Colossus. The secrets of Bletchley Park's codebreaking computers. Great Britain: Oxford University Press; 2006.

3. Viso E. Introducción a la teoría de la computación (Autómatas y lenguajes formales). México: Universidad Autónoma de México; 2008. Disponible en:

http://books.google.com.co/books/about/Introducc ion_a_la_Teoria_de_la_Computaci.html?id=NXQE8NJ w9d4C\&redir_esc $=y$

4. Bukachi F, Pakenham-Walsh N. Information technology of health in developing countries. Chest. 2007;132(5):1624-30.

5. Mosterín J. Racionalidad y acción humana. Madrid: Alianza Editorial SA; 2008.

6. Mosterín J. La naturaleza humana. Madrid, España: Grupo Planeta Spain; 2012. Disponible en: https://books.google.com.co/books?id=-

IkWpJQNq3YC\&dq=La+naturaleza+humana\&hl=es\& source=gbs_navlinks_s.

7. Lévy P. La cultura de la sociedad digital. Barcelona, España: Anthropos Editorial; 2007.

8. Fuchs C. Internet and society: social theory in the information age. New York: Routledge Research in Information Technology and Society; 2008. Disponible en: http://fuchs.uti.at/wpcontent/uploads/Internet+Society.pdf

9. Durkheim E. Las formas elementales de la vida religiosa. Madrid: Akal SA; 2007. Disponible en: https://books.google.com.co/books?id=MrPKiLWsX $\mathrm{hMC} \& \mathrm{dq}=\mathrm{Las}+$ formas+elementales+de+la+vida+reli giosa\&hl=es\&source=gbs_navlinks_s

10. Russell P. Peter Rusell, spirit of now. 2005. Disponible en: http://www.peterrussell.com/GB/GBAreview.ph

11. Lévy P. Inteligencia colectiva. Por una antropología del ciberespacio. Washington: OPS; 2004. Disponible en:

http://inteligenciacolectiva.bvsalud.org/public/doc uments/pdf/es/inteligenciaColectiva.pdf

12. Sparrow B, Liu J, Wegner D. Google Effects on Memory: Cognitive Consequences of Having Information at Our Fingertips. Science. 2011;333(6043):776-8.

13. Wegner D, Giuliano $T$, Hertel $P$. Cognitive Interdependence in Close Relationships. In: Psychology SSiS, editor. Compatible and incompatible Relationships. New York: Springer; 1985.

14. Educación Md. Educar. Argentina: Ministerio de 
Educación; 2010. [cited 2016 Mayo 17]. Disponible en: http://portal.educ.ar/debates/sociedad/elpoder-creciente-de-las-rede.php.

15. Dawkins R. El gen egoísta: las bases biológicas de nuestra conducta. Oxford : Salvat Editores; 2014.

16. Lankshear C. New Literacies: Changing Knowledge and Classroom Learning. Buckingham: Open University Press; 2003. Disponible en: https://books.google.com.co/books?id=Xg5NAEACAAJ\&dq=New+Literacies\&hl=es\&sa=X\&ei= r_lPVfniE7DnsASSIYHYBg\&ved=0CFIQ6 $w E w B w$

17. Burgess J. 'All Your Chocolate Rain Are Belong to Us' Viral Video, YouTube and the Dynamics of Participatory Culture. In: Cultures IoN, editor. Amsterdam: Video Vortex Reader: Responses to YouTube; 2008. p. 101-9.

18. Banco Mundial. Banco Mundial. Washington DC: Banco Mundial; 2015. Disponible en: http://datos.bancomundial.org/indicador/IT.NET.U SER.P2

19. Hull D. Taking memetics seriously: Memetic will be what we make it in Darwinizing Culture: the status of memetics as a Science. New York: Oxford University; 2003.

20. Clúa I. Genero y cultura popular. Estudios Culturales I. Barcelona: Ediciones Universidad Autónoma de Barcelona; 2008. [cited 2016 Mayo 16]. Disponible en: https://books.google.com.co/books?id=hxqeVodAwwC\&dq=G\%C3\%A9nero+y+cultura+popular. +Estudios+culturales\&hl=es\&source=gbs_navlinks_s

21. Aunger R. The Electric Meme: A New Theory of How We Think. New York: Simon and Schuster; 2013.

22. Huerta J. Socialismo, cálculo económico y función empresarial. Colombia: Unión Editorial; 2005. [cited 2016 Abril 30]. Disponible en: http://www.jesushuertadesoto.com/libros/librosen-espanol/socialismo-calculo-economico-yfuncion-empresarial/

23. Weber M. Economía y sociedad. México: Fondo de Cultura Económica; 1977.

24. Baylon C, Mignot X. La comunicación. Madrid: Gráficas Rogar SA; 1996.

25. Centola D, Baronchelli A. The spontaneous emergence of conventions: An experimental study of Cultural Evolution. Current Issue. 2014;112(7):1989-94.

26. Pisani F, Piotet D. La alquimia de las multitudes: Cómo la web está cambiando el mundo. Barcelona: Editorial Paídos Ibérica; 2009. [cited 2016 Abril 30]. Disponible en: http://books.google.com.co/books/about/La_alqui mia_de_las_multitudes.html?id=6Tp1oia9ekC\&redir_esc $=y$

27. Christakis N, Fowler J. The collective dynamics of smoking in a large social network. $\mathrm{N}$ Engl J Med. 2008;358:2249-58.

28. Suarez I. El gobierno de Internet. España: iSuarez; 2013. Disponible en
https://books.google.com/books?id=FcKvAQAAQBA J

29. Arstechnica. Arstechnica. California: Arstechnica; 2015. [cited 2016 Mayo 15]. Disponible en: http://arstechnica.com/science/2010/11/gametheory-explains-why-some-content-goes-viral-onreddit-digg/

30. Rifkin J. La sociedad del coste marginal cero: el Internet de las cosas, el procomún colaborativo y el eclipse del capitalismo. España: Editorial Paidós; 2014.

31. Ashton K. That 'Internet of Things' Thing. RFID Journal. 2011;2011:1.

32. Gartner. Gartner. Connecticut: Gartner; 2015. [cited 2016 Mayo 15]. Disponible en: http://www.gartner.com/technology/research/inte rnet-of-things/

33. Xataka. El Internet de las Cosas y la salud son parte de un nuevo futuro para BlackBerry. México: Xataka; 2015. [cited 2016 Mayo 20]; Disponible en: http://www.xataka.com.mx/eventos-detecnologia/el-internet-de-las-cosas-y-la-salud-sonparte-de-un-nuevo-futuro-para-blackberry

34. EuropeanCommmission. Green paper on mobile health. Bruselas: European Commission; 2014.

35. WHO. mHealth. New horizons for health through mobile technologies. Geneva: WHO; 2011.

36. OMS. La UIT y la OMS lanzan la Iniciativa salud móvil para luchar contra las enfermedades no transmisibles. Dubai: OMS; 2012. [cited 2016 Mayo 16]; Disponible en: http://www.who.int/mediacentre/news/releases/2 012/mHealth_20121017/es/

37. WHO. Accountability commission for health of women and children. Geneva: WHO; 2011. [cited 2016 Mayo 15]. Disponible en: http://www.who.int/topics/millennium_developme nt_goals/accountability_commission/en/

38. West D. How mobile devices are transforming healthcare. Issues in Technology Innovation, 2012;(18):1-14.

39. Ozdalga E, Ozdalga A, Ahuja N. The Smartphone in Medicine: A review of current and potential use among physicians and students. J Medical Internet R. 2012;14(5):e128.

40. IMS Institute for Healthcare Informatics. Patient adoption of mHealth. USA: IMS Institute for Healthcare Informatics; 2015. [cited 2016 Mayo 17]; Disponible http://www.imshealth.com/en/thoughtleadership/ims-institute/reports/patient-adoptionof-mhealth

41. TheAppDate. Informe 50 mejores Apps de salud en español. España: TheAppDate; 2014.

42. Lee J. Aplicaciones de salud diabética para dispositivos móviles: Exageración o esperanza. Atención sanitaria. 2014;59(3):43-6.

43. Organización Panamericana de la Salud. OPS lanza 
una aplicación para dispositivos móviles que ayuda a medir el riesgo de desarrollar una enfermedad cardiovascular. Washington: OPS; 2014. [cited 2016
Mayo
18].
Disponible
en:

http://www.paho.org/hq/index.php?option=com_c ontent\&view=article\&id=10022\%3A2014-paho-

launches-mobile-app-to-measure-cardiovascularrisk\&Itemid=1926\&lang=es

44. BlackBerry. BlackBerry for Healthcare. Canada: Black Berry; 2016. [cited 2016 Mayo 20]; Disponible en:

http://global.blackberry.com/en/enterprise/indust ries/healthcare.html

45. Apple. La forma más innovadora de usar tu información de salud y bienestar. USA: Apple; 2016. [cited 2016 Mayo 19]. Disponible en: http://www.apple.com/co/ios/health/

46. Valle S. Cibercultura y civilización universal: Hacia un nuevo orden cultural. Barcelona: Erasmus Ediciones; 2011. 